\title{
Reconstruction of Aesthetic Stomatognathic Function After a High-energy Maxillo-orofacial Fracture: a Case Report
}

\section{Toshiyuki Kataoka ( $\nabla$ kataoka.toshiyuki@twmu.ac.jp )}

Department of dentisty and oral surgery https://orcid.org/0000-0001-6042-4014

\section{Kei Amemiya}

Tokyo Women's Medical University Yachiyo Medical Center: Tokyo Joshi Ika Daigaku Yachiyo Iryo Center

\section{Gen Udagawa}

Tokyo Women's Medical University Yachiyo Medical Center: Tokyo Joshi Ika Daigaku Yachiyo Iryo Center

\section{Yuichi Akagi}

Tokyo Women's Medical University: Tokyo Joshi lka Daigaku

\section{Chie Kagawa}

Tokyo Women's Medical University: Tokyo Joshi lka Daigaku

\section{Toshihiro Okamoto}

Tokyo Women's Medical University: Tokyo Joshi Ika Daigaku

\section{Case report}

Keywords: High-energy injury, maxilla-orofacial fracture, fixed prosthesis, osseointegrated implant

Posted Date: December 28th, 2020

DOl: https://doi.org/10.21203/rs.3.rs-133150/v1

License: (a) (1) This work is licensed under a Creative Commons Attribution 4.0 International License. Read Full License 


\section{Abstract}

Background: High-energy injury patients with head trauma often present with pan-jaw fractures involving loss of teeth and alveolar bone. They experience a significant decrease in their quality of life due to masticatory disorders, dysarthria, and poor aesthetics. In most cases, they are treated separately by a medical team and a prosthodontist. Moreover, removable dentures provide little satisfaction to the patients.

Case presentation: A 29-year-old young woman suffered a high-energy trauma from a fall accident. Following surgery for a jaw fracture, she underwent occlusal reconstruction with osseointegrated implants. The same team performed maxillofacial surgery and prosthetic treatments, and the series of treatment plans included bone grafting and periodontal plastic surgery.

Conclusions: The treatment proved successful after more than two years with no signs of peri-implantitis. The patient was highly satisfied with the results. Early intervention by an oral and maxillofacial surgeon in such patients is essential for a good prognosis. Surgeons and prosthodontists who belong to the same team could complete the treatment with fewer surgical procedures. Furthermore, implant treatment in a patient with oral and maxillofacial fractures might improve aesthetics and function.

\section{Background}

Patients who undergo surgery for maxillofacial high-energy trauma (HET) may have facial deformities, bone healing failures, jaw dysfunctions, and malocclusions. Additionally, teeth or alveolar bone defects result in masticatory disorders, dysarthria, and poor aesthetics, delaying the patient's social rehabilitation and significantly reducing their postoperative quality of life (QOL). Therefore, it is important to achieve symmetric facial morphology and reliable occlusion reconstruction.

Restoring morphology and function using conventional, removable dentures is challenging. Maxillofacial implants are required for predictable occlusal reconstruction in patients with maxillofacial trauma. The periodontal tissue environment around the implant affects its long-term stability; therefore, a treatment plan involving bone augmentation (alveolar ridge and GBR) and periodontal plastic surgery is required. Owing to the presence of considerable expectations for the results and the associated process, it is imperative to provide a detailed diagnosis of the condition to maxillofacial and oral surgeons treating HET jaw and occlusal reconstruction.

We present a reconstruction case using a fixed prosthesis and osseointegrated implants after maxillofacial trauma due to HET. A bone graft was necessary for dental implantation following the treatment of the jaw fracture. Digital data and setup models were useful for treatment planning. The patient was highly satisfied with the results of the treatment.

\section{Case Presentation}


A 29-year-old woman accidentally fell from a height of three floors at a construction site. It took an hour to rescue her from the site, and then she was brought to our emergency department for advanced trauma life support. She was fortunate to have no significant intracranial, spinal, or viscerotropic injuries. There were extensive abrasions and a subcutaneous hemorrhage on the face. The right mid-third of the face had recessed, and there was a deep laceration in the lower jaw. The frontal wall of the maxillary sinus was shattered and depressed, and the maxillary dentition was displaced upward and inward, resulting in loss of continuity. Furthermore, the alveolar bone in the upper right 13-17 region presented with a comminuted fracture. Teeth 13,15 , and 16 were located within the fractured area. There was an insular depressed fracture in the right mandible, and the lower right 42-46 dentition were displaced inwards, resulting in an open bite due to early occlusal contacts of the molars. There was a the right inferior alveolar nerve paralysis. (Figs. 1 and 2).

We confirmed the preoperative and postoperative dentition and occlusal conditions before surgery via model surgery and formulated a surgical plan. The first surgery was performed under general anesthesia, and the dentition was restored as planned. There was no depressed fracture in the zygomatic arch; therefore, the maxillary dental arch, the anterior wall of the maxillary sinus, and the richness of the cheek were restored. The dislocated upper right canine was fixed within the dentition, whereas teeth 15 and 16 were abandoned due to the crushed alveolar bone and the maxillary sinus's anterior wall. The maxillary first premolar had previously been extracted through an orthodontic procedure. The mandibular fracture underwent open reduction and fixation using a titanium plate (Figs. 3A, B).

The second surgery for implant removal and maxillary alveolar bone defect atrophy was planned for six months after the first surgery. Occlusal reconstruction was initiated using digital data and a 3D molded model to envision a fixed prosthesis with an osseointegration implant prior to surgery. A cross-sectional computed tomography (CT) scan revealed that the fractured roots of tooth 16 were lodged within the fractured bone and could interfere with placing the dental implant. The alveolar bone in the dentition defect had insufficient height and width for dental implantation. The missing 15 and 16 atypical spaces were recovered using two premolar-shaped prostheses according to the outcomes of diagnostic setup. In The 3D model, it was clear that the alveolar bone in the dentition defect was atrophied and lacked in height and width.(Figs. 4 and 5). An osteotomy of the mentum, from which the titanium plate was removed, was performed $(40 \times 20 \mathrm{~mm})$; half of it was veneer-grafted with a cortical bone block. The other half was crushed and used to fill the gap to GBR using a titanium mesh (Fig. 6A, B, C). The amount of horizontal bone width created was deemed sufficient; additionally, there were no complaints of any abnormal sensations in the anterior teeth in the mandible following bone collection.

The patient underwent a third operation six months later. Due to trauma and the previous surgery, the scar on the tooth defect's alveolar mucosa was visible, and the oral vestibule was narrowed. Non-mobile keratinized mucosa were required in the alveolar ridge mucosa of the dentition. Thus, vestibuloplasty using atelocollagen was performed when the titanium mesh was removed. Two dental implants (Xive dental implants from DENTSPLY, Mannheim GERMANY) were placed on the alveolar ridge after the healing of the mucosa (4 months). A surgical procedure was performed three months later, wherein the 
attached gingiva at the alveolar crest was extended to the oral vestibular side to obtain keratinized gingiva around the dental implant (Fig. 7).

The provisional restoration was used to harmonize the crown form and gingival morphology, and the final superstructure was attached about three years after the injury. The patient was fully satisfied with the aesthetic and functional properties. She responded to a maintenance system every 4-6 months and has maintained her oral hygiene with no signs of peri-implantitis (Fig. 8).

\section{Discussion And Conclusion}

In severe maxillofacial injury due to HET, prompt cooperation between the primary survey and the specialized departments concerned with the adjacent affected organs is required. In cases where specialized diagnosis and treatment are required to manage systemic and oral/ maxillofacial injuries, it is beneficial to include an oral and maxillofacial during the treatment planning ${ }^{1)}$. Multiple maxillofacial injuries result in loss of alveolar mucosa in addition to jaw bone fractures and missing or damaged teeth. Dental implants are effective in restoring the aesthetic and oral functions ${ }^{2,3)}$. However, the reconstruction process requires a long treatment period and many surgeries. There are several advantages of including a maxillofacial surgeon for the occlusal reconstruction during the treatment planning. In the absence of any issues with life support, it is important to consult with adjacent medical specialists, such as brain surgeons, ophthalmologists, and otolaryngologists to start the diagnosis and treatment planning for the middle and lower thirds of the face. The major goals of treating maxillofacial fractures are to restore the midface's symmetry and the mandible's continuity. It is important to follow evidence-based treatment strategies ${ }^{4)}$ for good results with maxillofacial fracture treatment.

Occlusal reconstruction is the main theme of stomatognathic medicine. It begins 6 months after mandibular fracture following confirmation of the absence of disorders in the temporomandibular joint. It is desirable for this medical team to be able to organically collaborate with the oral surgery team responsible for jawbone fractures, the prosthetic team for restoring the dentition and occlusion, and the periodontal team for improving the periodontal environment. Dental implant treatment aims to match morphological aesthetics with functionality. In order to achieve the maximum effects of the implant in terms of occlusal reconstruction, it is important to assess the condition of the periodontal tissues and place the osseointegrated implant in the appropriate position ${ }^{5)}$. Therefore, the use of 3D digital images is useful during treatment planning.

The minimum bone width and height of the alveolar ridge for dental implantation must be $>5 \mathrm{~mm}$ and $>$ $10 \mathrm{~mm}$, respectively ${ }^{6}$ ). Reports on the use of distraction osteogenesis, autologous bone grafting ${ }^{7)}$, titanium mesh tray, and iliac particulate cancellous bone and marrow transplantation (Ti-MESH method) ${ }^{8,9)}$ for bone defects have been published in the literature. The advantage of distraction osteogenesis is that the soft tissue can concurrently be expanded; however, the dynamic treatment period of several months is a major drawback. In the Ti-MESH method, the biggest concern is the surgical invasion of the ilium crest. Expected results may not be obtained due to the titanium mesh's adverse events (tray 
exposure and increased risk of infection) and remodeling resorption, leading to loss of the graft ${ }^{10,11)}$. Bone grafting is considered optimal for complex and extensive bone loss due to trauma. Autologous bone grafting, which has excellent bone formation ability, is the gold standard for bone regeneration. Since the donor site's structure and anatomical site may affect the outcome of the bone graft ${ }^{12}$ ), the recipient site should be closer to the donor if possible. Bone graft planning requires a $10-20 \%$ increase in target bone regeneration owing to the resorption of the graft bone. ${ }^{13)}$. It has been reported that the amount of the grafted bone absorbed is less in the mandible generated through intramembranous ossification than in the iliac crest generated through endochondral ossification. ${ }^{14,15)}$. The chin bone is highly calcified, which helps to make an early transition to the mandible ${ }^{16)}$. In addition, the cortical bone with its high mineral density is useful for providing the primary stability for the dental implants. We are selected the recipient sites according to the requirements of the donor. In the this case study, the chin's cortical bone was selected because it could be collected from the same surgical field. The use of chin cortical bone may be useful when grafting a bone to address a few alveolar bone defects.

The keratinized mucosa in the alveolar ridge is often lost along with the alveolar bone following a jaw fracture. Alternatively, a scarred mucosa remains after several surgical procedures. The mobile nonkeratinized mucosa around the dental implant is more likely to cause peri-implantitis ${ }^{17)}$. Also, the development of a mucosal scar with poor blood flow is disadvantageous for bone formation. The presence of keratinized mucosa around the dental implants affects the long-term prognosis of the implant treatment ${ }^{18,19}$. Therefore, vestibuloplasty and free gingival grafts to acquire a keratinized mucosa ${ }^{20)}$ are required. The palatal mucosa or atelocollagen is effective as a recipient of mucosal grafts 21,22). It is important to ensure that a keratinized mucosa (of $2 \mathrm{~mm}$ ) is present around the dental implant. An oral photograph is indispensable because it is impossible to evaluate the soft tissues using X-ray photography or models. At present, it is unclear when the periodontal mucosa should be treated. The apically positioned flap technique is often performed at the same time as the secondary operation. However, oral mucosal defects after maxilla-orofacial trauma may require mucosal management before implant placement.

For patients with severe facial fractures, strict adherence to a well-established and structured treatment protocol based on surgical experience provides an efficient, appropriate, and successful treatment. Furthermore, superior results for severe traumatic maxilla-orofacial injuries will be achieved if the treatment is combined with efficient occlusal reconstruction.

\section{Abbreviations}

HET: high-energy trauma

QOL: quality of life

GBR: Guided Bone Regeneration 


\section{Declarations}

- Ethics approval and consent to participate

This study was conducted following the World Medical Association's ethical guidelines, as described in the Declaration of Helsinki released in 2013.

- Consent for publication

The patient provided written informed consent for publication of this case report and any accompanying images.

- Availability of data and materials

The datasets generated and/or analyzed during the current study are not publicly available due to inconsistent language expression but are available from the corresponding author upon reasonable request

- Competing interests

The authors declare that they have no competing interests.

- Funding

This research did not receive any specific funding from public, commercial, or not-for-profit agencies.

- Authors' contributions

All authors have made substantial contributions to the conception and design of the study. KA and GU have been involved in data collection, YA and CK have been involved indata analysis, TK and TO have been involved in data interpretation. TK drafted the manuscript. All authors revised it critically and have given final approval of the version to be published. All authors read and approved the final manuscript.

- Acknowledgments

The authors would like to thank Enago (www.enago.jp) for the English language review.

\section{References}

1. Kanno T, Sukegawa S, Nariai Y, Tatsumi H, Ishibashi H, Furuki Y, et al. Surgical treatment of comminuted mandibular fractures using a low-profile locking mandibular reconstruction plate system. Ann Maxillofac Surg. 2014;4:144-9.

2. Ormianer Z, Piek D, Livne S, Lavi D, Zafrir G, Palti A, et al. Retrospective clinical evaluation of tapered implants: 10-year follow-up of delayed and immediate placement of maxillary implants. Implant 
Dent. 2012;21:350-6.

3. Pjetursson BE, Asgeirsson AG, Zwahlen M, Sailer I. Improvements in implant dentistry over the last decade: comparison of survival and complication rates in older and newer publications. Int $\mathrm{J}$ Oral Maxillofac Implants. 2014;29 Suppl:308-24.

4. Audigé L, Cornelius CP, leva AD, Prein J, CMF Classification Group. The first AO classification system for fractures of the craniomaxillofacial skeleton: rationale, methodological background, developmental process, and objectives. Craniomaxillofac Trauma Reconstr. 2014;7:S006-14.

5. Gellrich NC, Rahlf B, Zimmerer R, Pott PC, Rana M. A new concept for implant-borne dental rehabilitation; how to overcome the biological weak-spot of conventional dental implants?Head Face Med. 2017;13:17.

6. Kataoka T, Akagi Y, Kagawa C, Sasaki R, Okamoto T, Ando T. A case of effective oral rehabilitation after mandibular resection Clin Case Rep. 2019;7:2143-48.

7. Aghaloo TL, Moy PK. Which hard tissue augmentation techniques are the most successful in furnishing bony support for implant placement? Int J Oral Maxillofac Implants. 2007:22 Suppl:49-70.

8. lino $\mathrm{M}$, Shimizu $\mathrm{H}$, Kasahara $\mathrm{H}$. Alveolar ridge augmentation using autogenous iliac cancellous bone graft combined with Micro-Titanium Augmentation Mesh and subsequent dental implant insertion. Asian J Oral Maxillofac Surg. 2000;12:187-94.

9. Yamada H, Nakaoka K, Horiuchi T, Kumagai K, Ikawa T, Shigeta Y, et al. Mandibular reconstruction using custom-made titanium mesh tray and particulate cancellous bone and marrow harvested from bilateral posterior ilia. J Plast Surg Hand Surg. 2014;48:183-90.

10. Louis PJ, Gutta R, Said-Al-Naief N, Bartolucci AA. Reconstruction of the maxilla and mandible with particulate bone graft and titanium mesh for implant placement. J Oral Maxillofac Surg. 2008;66:235-45.

11. Kumar BP, Venkatesh V, Kumar KA, Yadav BY, Mohan SR. Mandibular reconstruction: overview. J Maxillofac Oral Surg. 2016;15:425-41.

12. Hassani A, Khojasteh A, Shamsabad AN. The anterior palate as a donor site in maxillofacial bone grafting: A quantitative anatomic study. J Oral Maxillofac Surg. 2005;63:1196-200.

13. Aghaloo TL, Moy PK. Which hard tissue augmentation techniques are the most successful in furnishing bony support for implant placement? Int J Oral Maxillofac Implants. 2007;22(Suppl):4970.

14. Koole R, Bosker $\mathrm{H}$, van der Dussen FN. Late secondary autogenous bone grafting in cleft patients comparing mandibular (ectomesenchymal) and iliac crest (mesenchymal) grafts. J Craniomaxillofac Surg. 1989;17:28-30.

15. Mish CM, Mish CE, Resnik RR, Ismail YH. Reconstruction of maxillary alveolar defects with mandibular symphysis grafts for dental implants: a preliminary procedural report. Int $\mathrm{J}$ Oral Maxillofac Implants. 1992;7:360-6.

16. Garg AK. Dental implants for the facial truma patients. Dental Implantol Update 1998;9:25-9. 
17. Gobbato L, Avila-Ortiz G, Sohrabi K, Wang CW, Karimbux N. The effect of keratinized mucosa width on peri-implant health: a systematic review. Int J Oral Maxillofac Implants. 2013;28:1536-45.

18. Gharpure AS, Gharpure AS, Patankar NM. Significance of keratinized tissue assessment in implant therapy. Dentist Case Rep. 2018;1:5-7.

19. Ladwein C, Schmelzeisen R, Nelson K, Fluegge TV, Fretwurst T. Is the presence of keratinized mucosa associated with periimplant tissue health? A clinical cross-sectional analysis. Int J Implant Dent. 2015;1:11.

20. Padhye NM, Mehta LK, Yadav N. Buccally displaced flap versus sub-epithelial connective tissue graft for peri-implant soft tissue augmentation: a pilot double-blind randomized controlled trial. Int $\mathrm{J}$ Implant Dent. 2020;6:48.

21. Tavelli L,Barootchi S,Ravidà A, Oh TJ, Wang HL. What Is the Safety Zone for Palatal Soft Tissue Graft Harvesting Based on the Locations of the Greater Palatine Artery and Foramen? A Systematic Review. J Oral Maxillofac Surg. 2019;77:271.e1-271.e9.

22. Xu C, Wang QT, Chen J, Wu Y, Zhao L. Collagen matrix for periodontal plastic surgery procedures: a meta-analysis update. Int J Periodontics Restorative Dent. 2019;39:e129-e155.

\section{Figures}

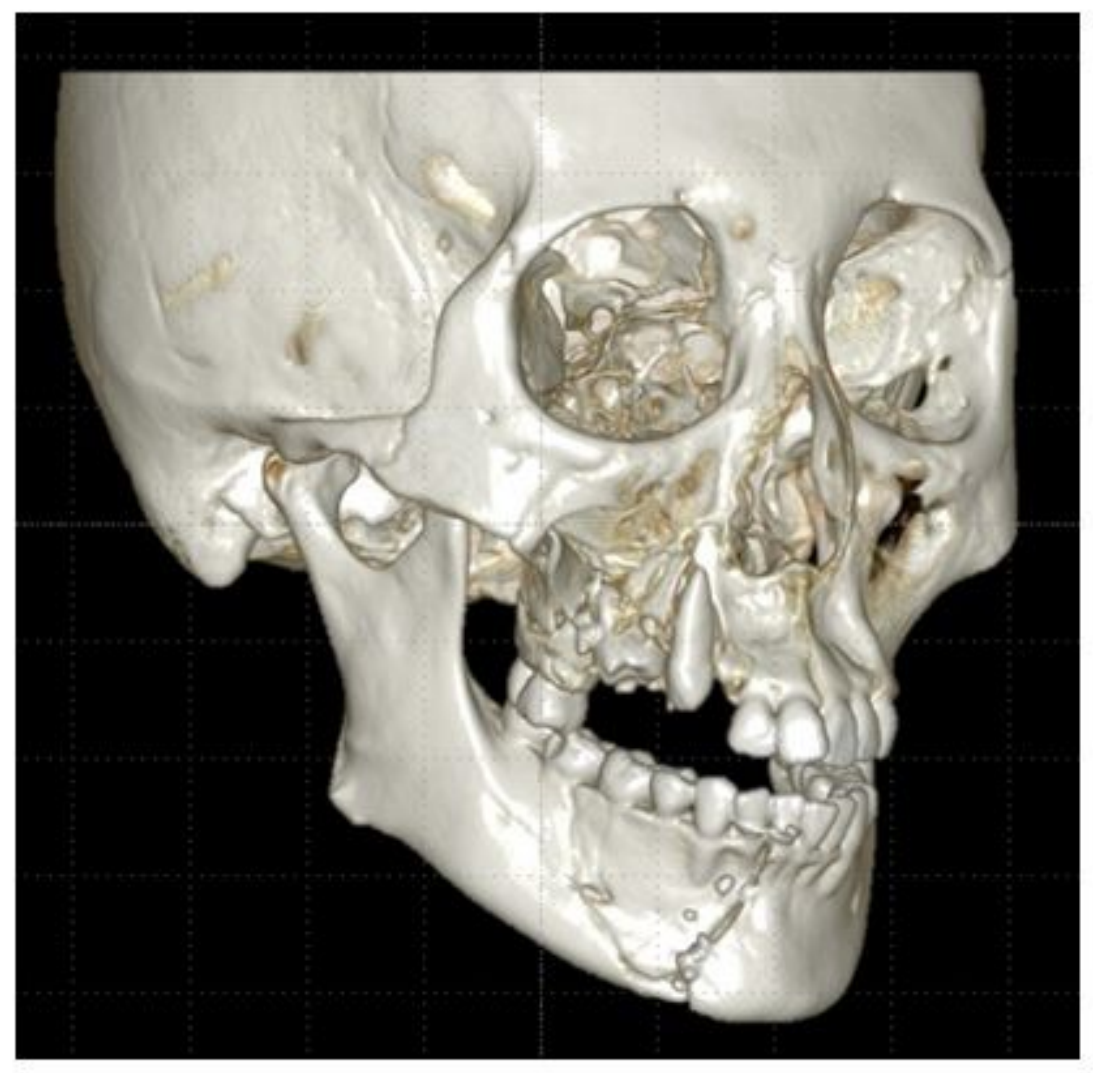

Figure 1 
Facial 3DCT image at the time of initial treatment. The right maxilla has a crushed fracture. The upper right canine is dislocated and invaginated, and the upper right second premolar and first molar are invaded into the crushed bone. The right mandible has a depressed fracture, and the fracture line crosses the mental foramen.

A

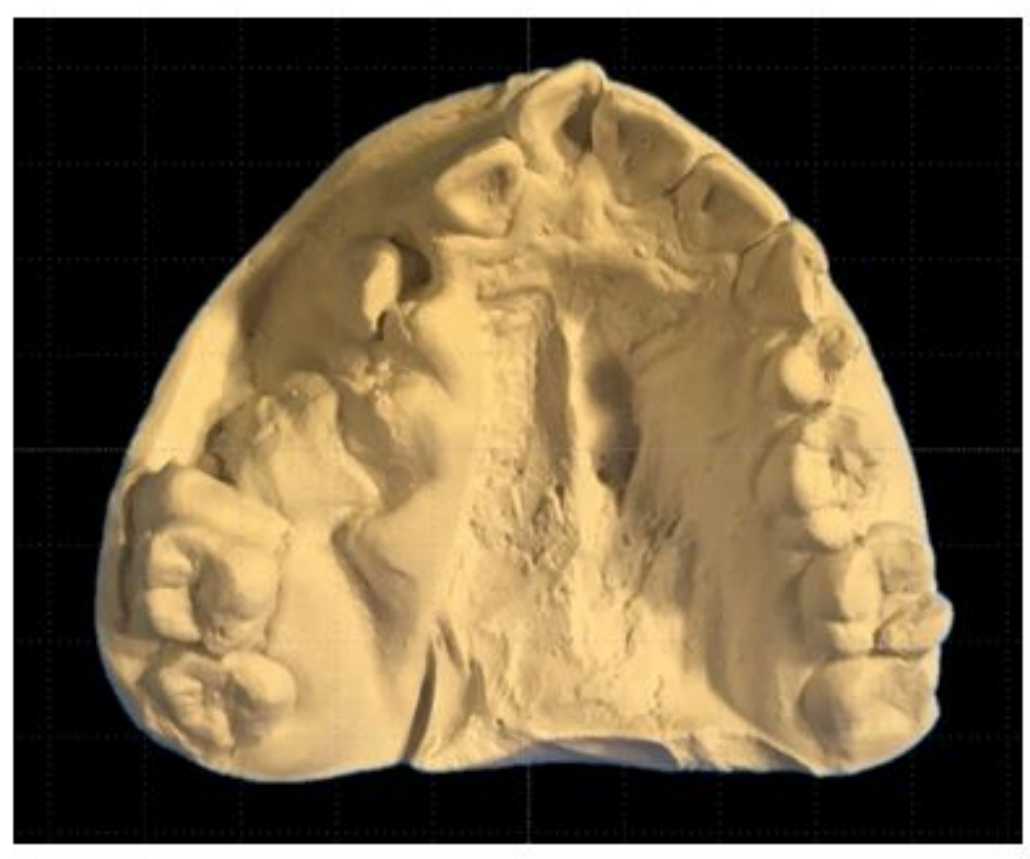

B

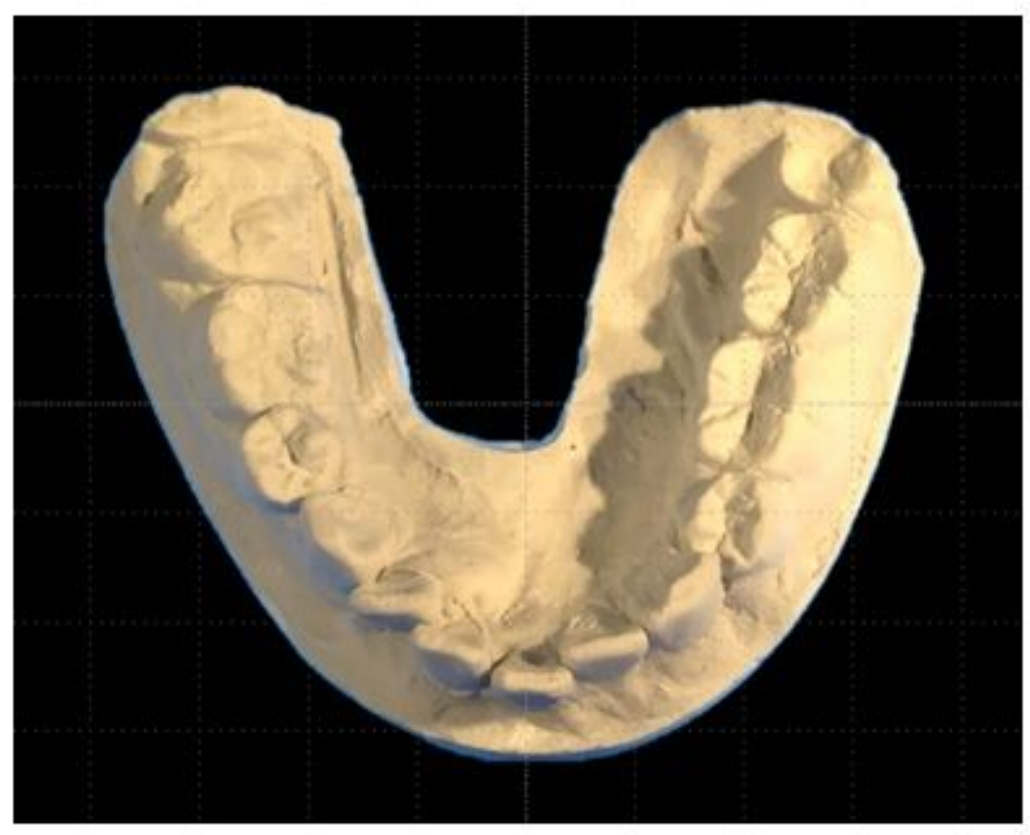

\section{Figure 2}

Examination models of the upper and lower dentitions. The right maxillary dentition is displaced medially with loss of continuity. The upper right canine is dislocated, and the second premolar and first molar have 
deviated from the dentition. The right mandibular dentition is laterally displaced. Model surgery was performed before the first surgery to clarify the posttreatment dentition imaging.

A

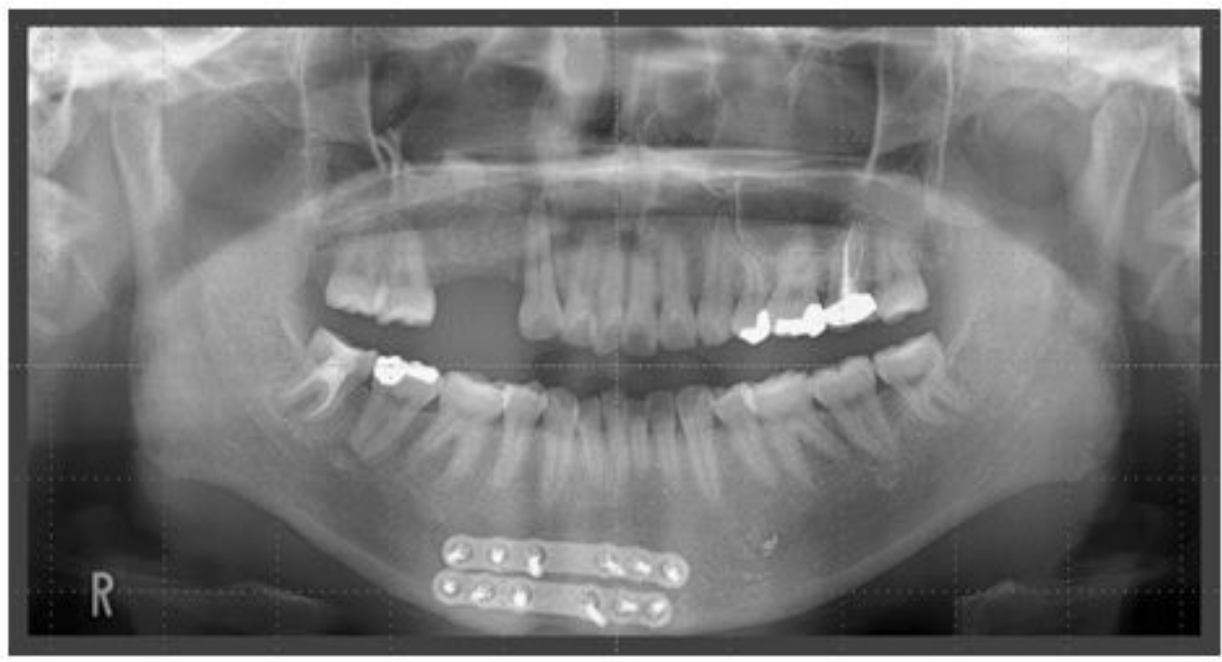

B

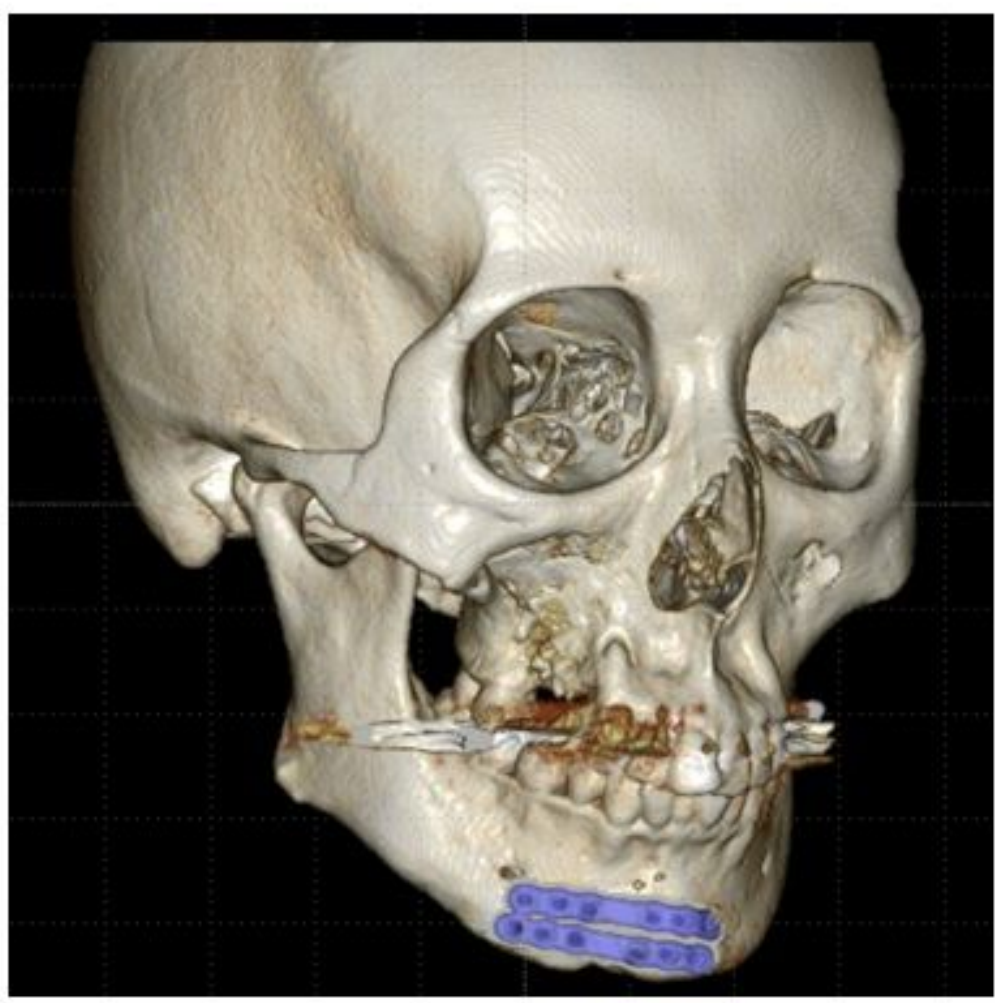

\section{Figure 3}

Radiographic images were obtained 6 months after open reduction and fixation. A. Orthopantomograph showing the reduction of the upper right canine and its retention within the dentition. B. 3DCT photograph demonstrating the healing of the fracture line after reduction and fixation with two titanium plates. 
A

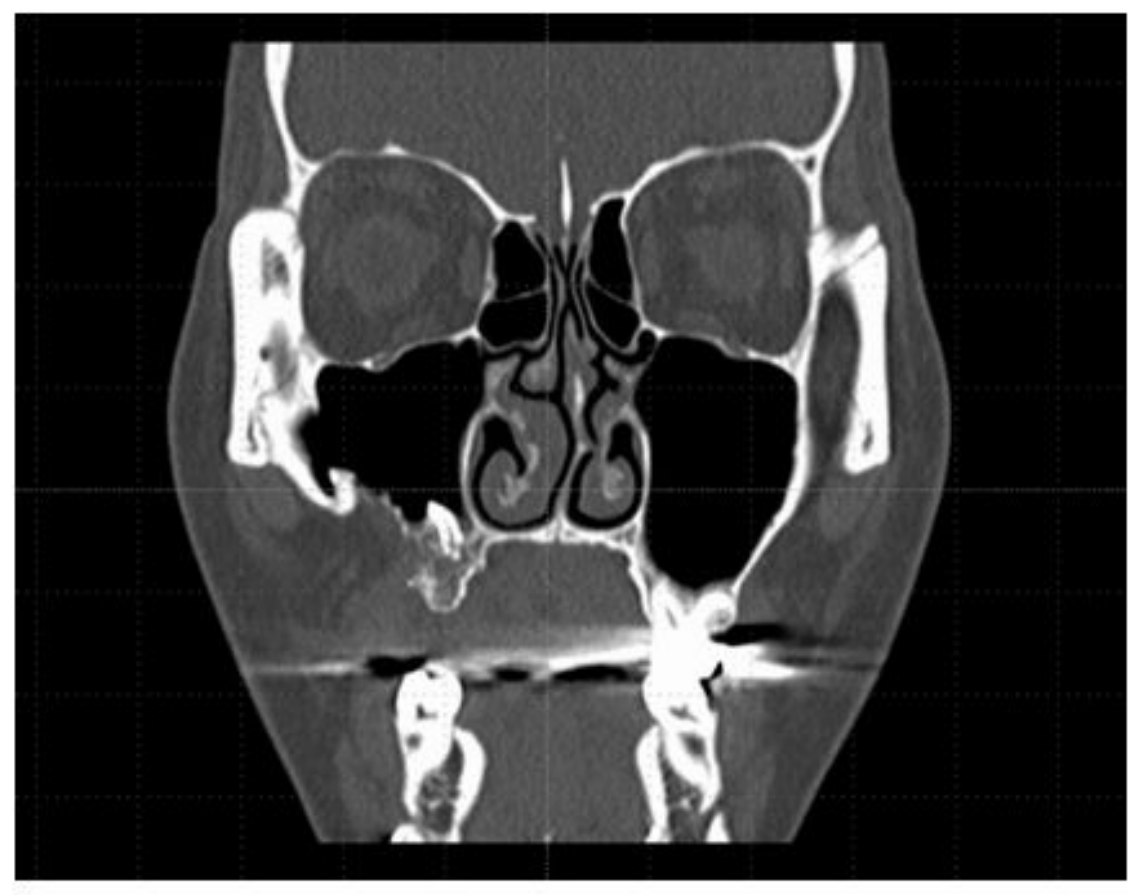

B

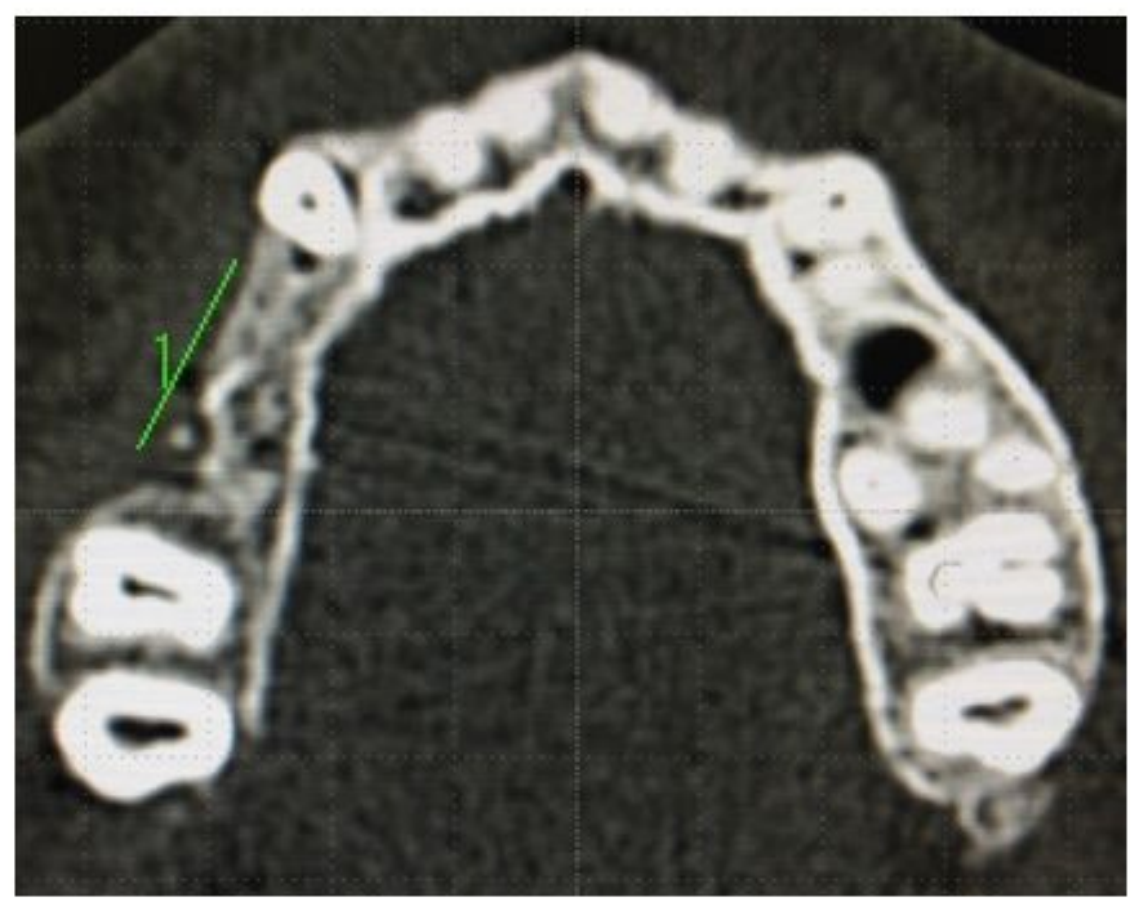

Figure 4

CT views of the jaws. A. The coronal view shows that the fractured root of the upper right first molar within the bone is retained. The amount of alveolar bone required for dental implant placement is insufficient in terms of both height and width. B. In the axial view, the length of the alveolar at the tooth loss was atypical. It was revealed that the premolars on the opposite side had 2.5 to 3 teeth. 
A

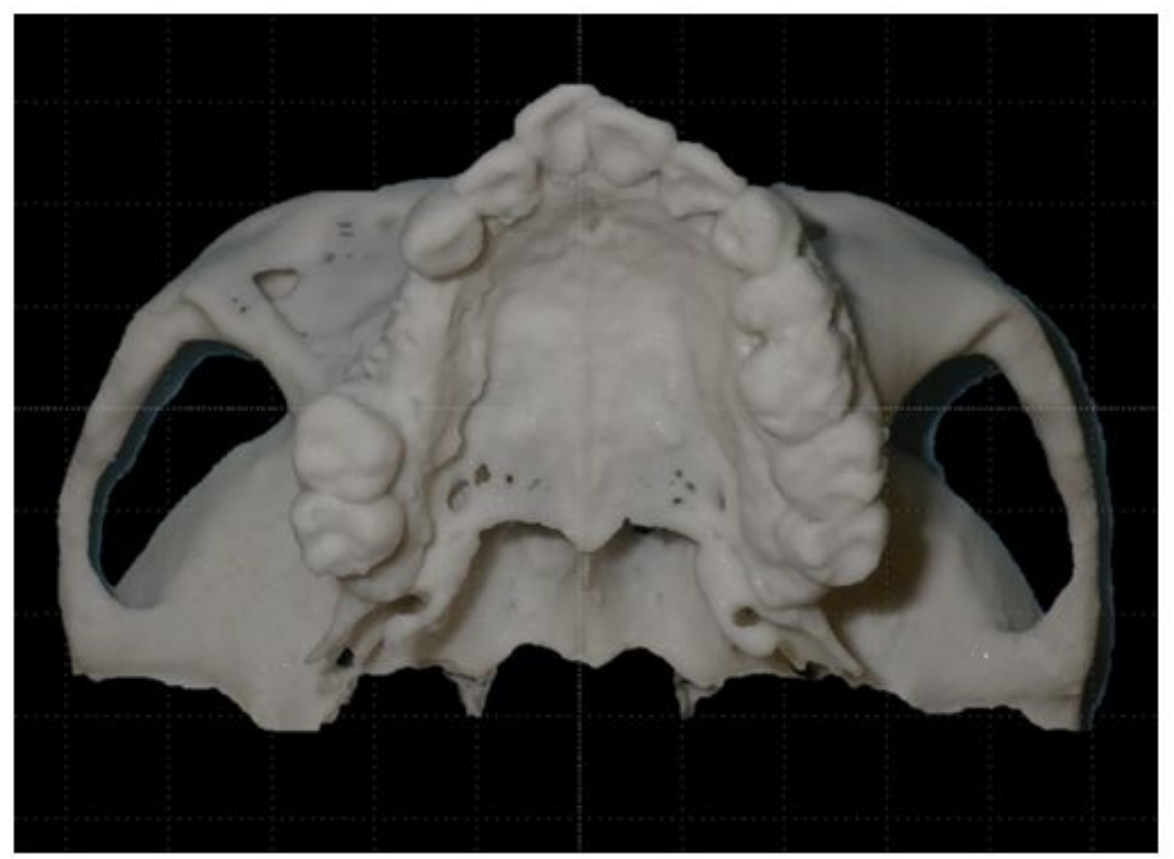

B

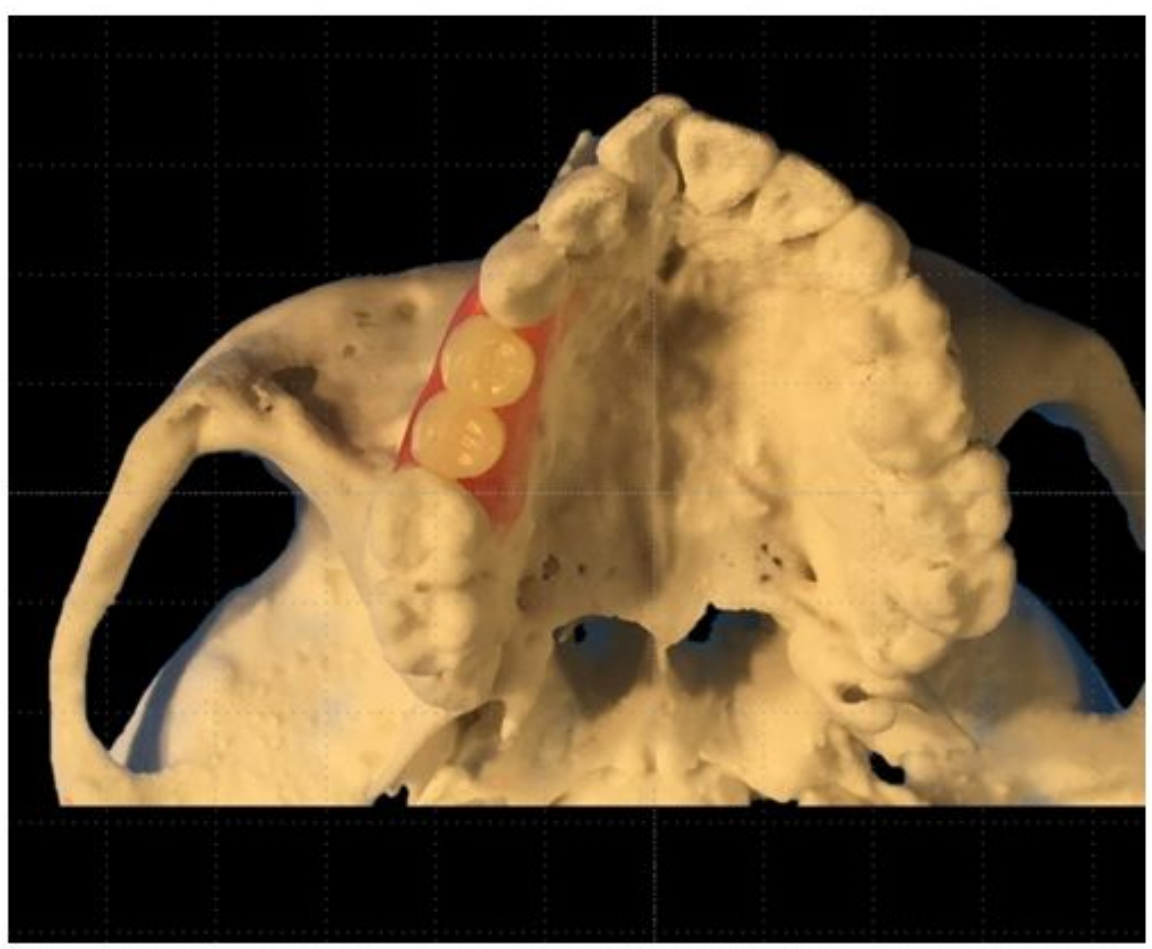

Figure 5

The 3D model. A. The maxillary 3D model's occlusal view shows that the alveolar ridge of the upper right premolar is narrow, and implants cannot be placed. B. Simulation of periodontal tissue in harmony with the prosthesis. The insufficient height and width of the periodontal tissue in the premolar region are clear. The size of the prosthesis was decided to be equal to that of two large premolars. 
A

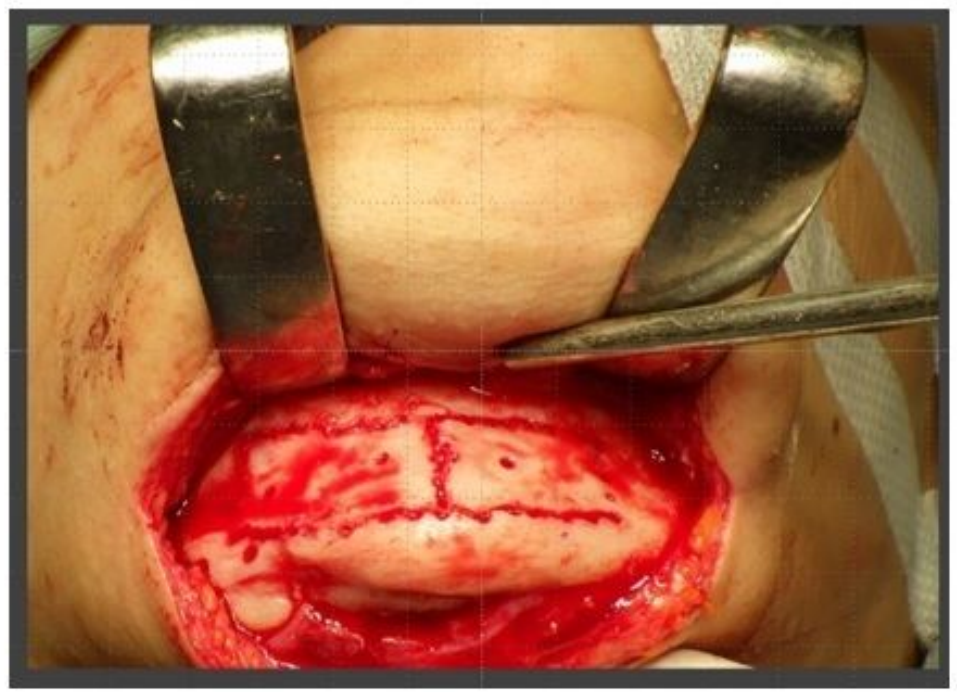

C

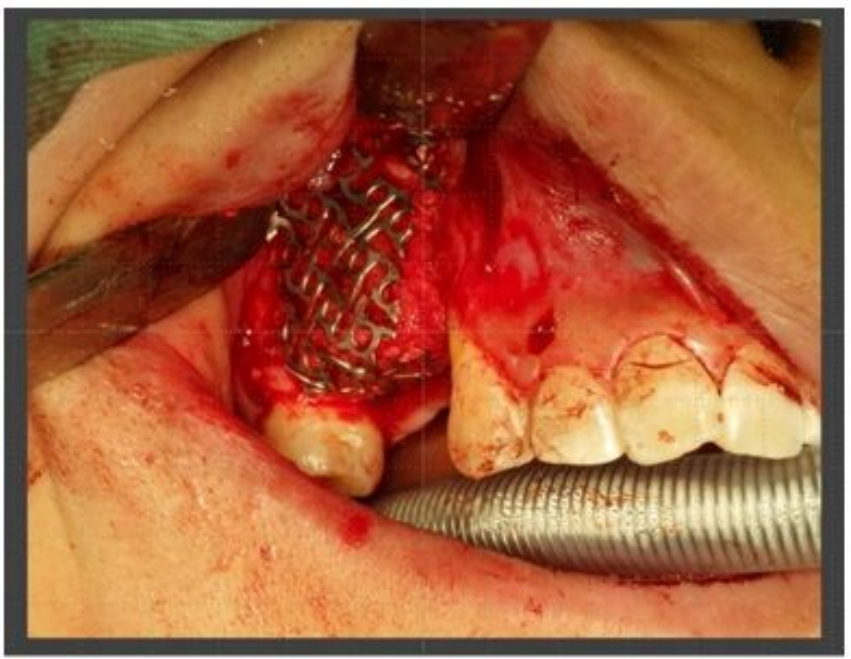

B

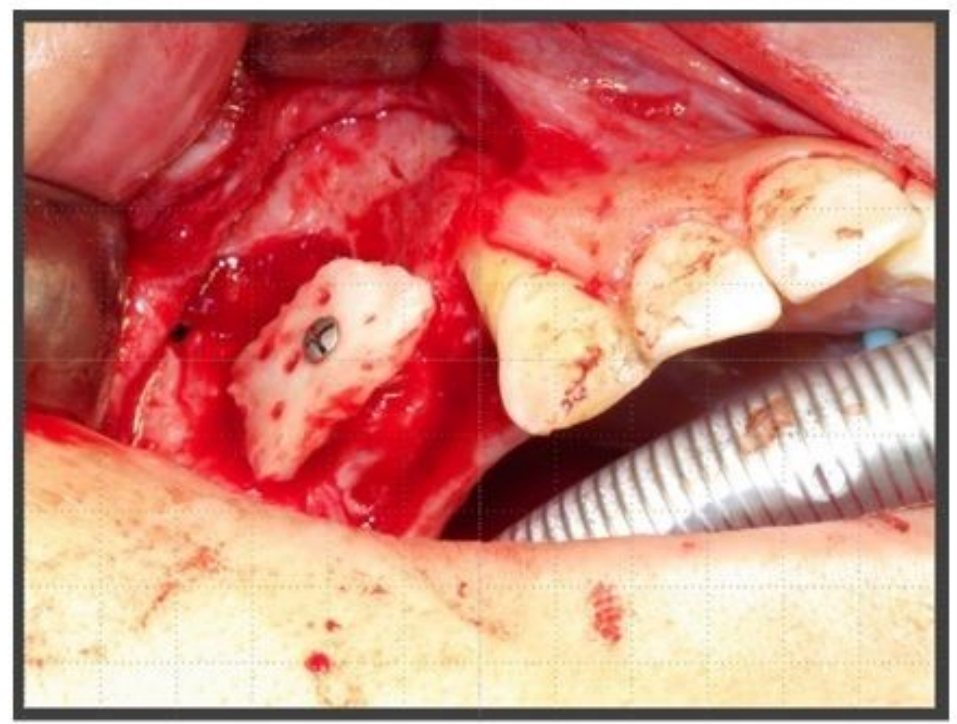

Figure 6

Surgerical view at the time of bone graft A. Surgical field from which the titanium plate that was used to fix the mandibular fracture was removed. The cortical bone of the chin was osteotomized at $40 \times 20 \mathrm{~mm}$. B. One cortical bone $(20 \times 20 \mathrm{~mm})$ was veneer-grafted to provide bone width and height. C. The other graft was crushed and filled within the veneer graft gaps and shaped with a titanium mesh. 
A

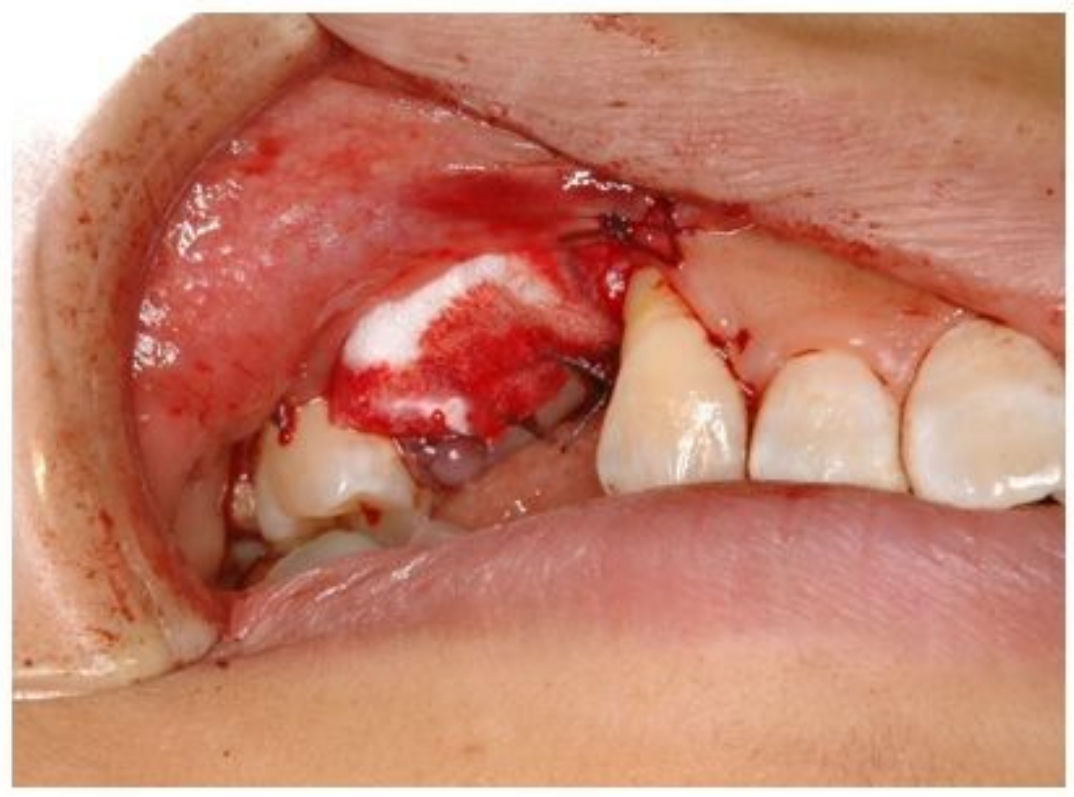

B

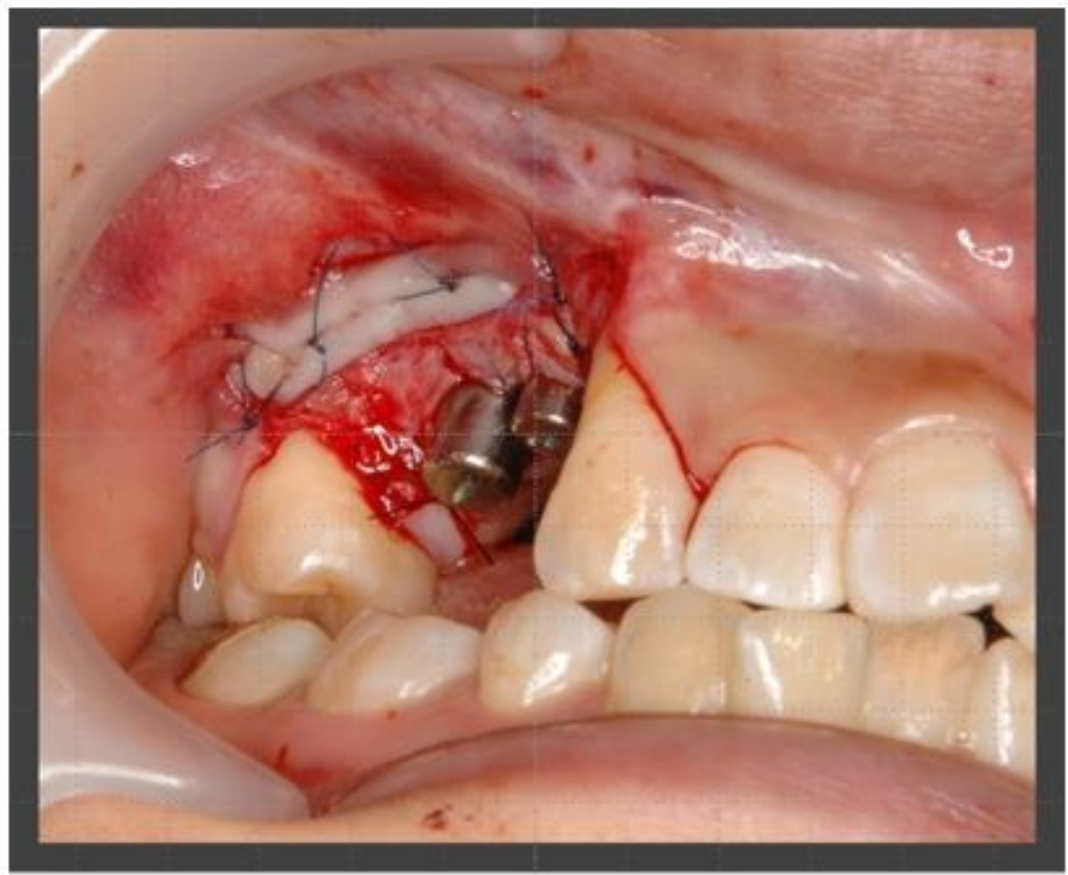

Figure 7

Periodontal plastic surgery was performed twice to obtain the attached keratinized mucosa. A. Vestibuloplasty using atelocollagen was performed at the same time when the titanium mesh was removed. B. The keratinized alveolar mucosa was moved apically when the second procedure was performed. 
A

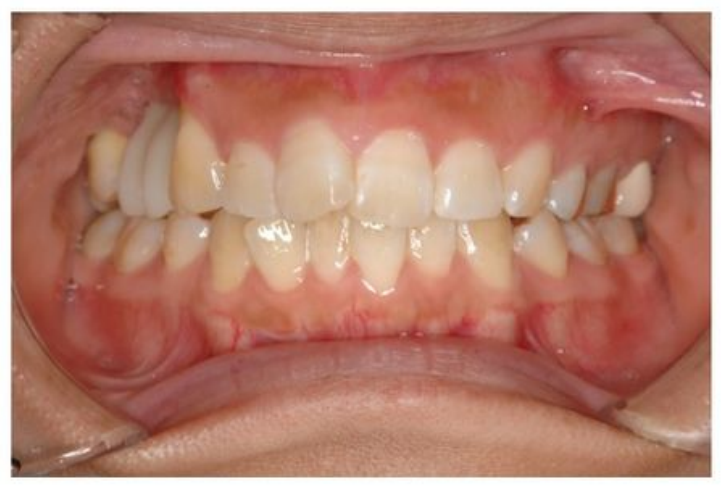

B

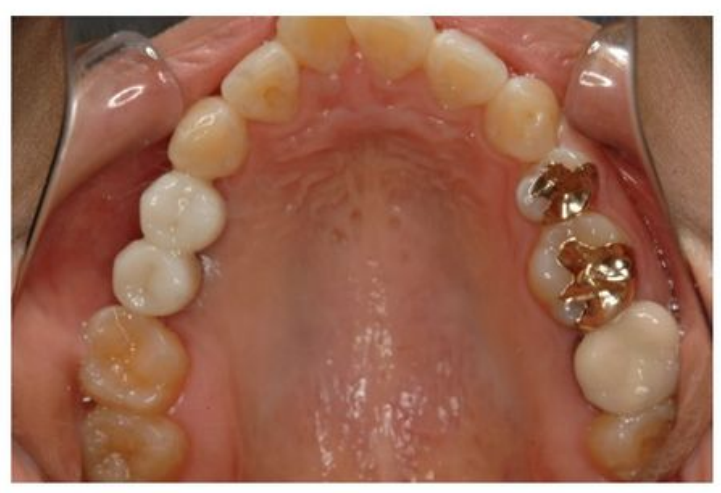

$\mathrm{C}$

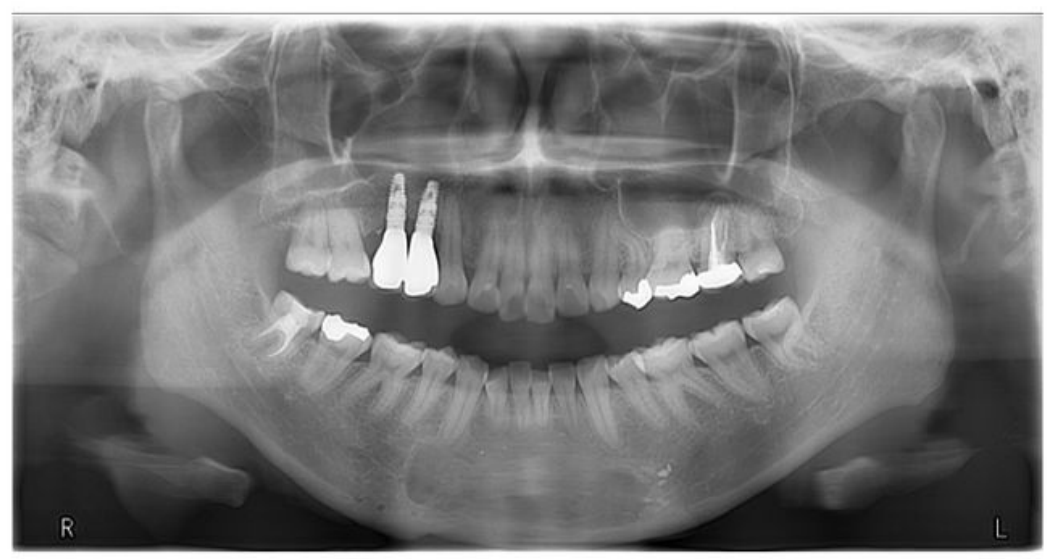

\section{Figure 8}

Results of the treatment. A. Image showing the intraoral frontal view. B. Image showing the intraoral occlusal view. The final prosthesis was fitted according to the treatment plan. The fixed implant prosthesis was supported by the keratinized mucosa and maintained in a favorable environment. Two years have passed since the last prosthesis was attached, and no peri-implantitis was observed. C. Orthopantomograph 2 years after the superstructure was attached. No bone resorption was observed around the implant body.

\section{Supplementary Files}

This is a list of supplementary files associated with this preprint. Click to download.

- CAREchecklistEnglish2013.docx 